Published in final edited form as:

Curr Opin Cardiol. 2017 November ; 32(6): 761-766. doi:10.1097/HCO.0000000000000445.

\title{
Gut Microbiome and its Role in Cardiovascular Diseases
}

\author{
Shadi Ahmadmehrabi ${ }^{1}$ and W. H. Wilson Tang, $\mathbf{M D}^{2,3}$ \\ ${ }^{1}$ Cleveland Clinic Lerner College of Medicine at Case Western Reserve University, Cleveland, \\ Ohio \\ ${ }^{2}$ Heart and Vascular Institute, Cleveland Clinic, Cleveland, Ohio \\ ${ }^{3}$ Center for Clinical Genomics, Cleveland Clinic, Cleveland, Ohio
}

\begin{abstract}
Purpose of Review-In recent years, an interest in intestinal microbiota-host interactions has increased due to many findings about the impact of gut bacteria on human health and disease. Dysbiosis, a change in the composition of the gut microbiota, has been associated with much pathology, including cardiovascular diseases (CVD). This article will review normal functions of the gut microbiome, its link to CVD, and potential therapeutic interventions.
\end{abstract}

Recent findings-The recently discovered contribution of gut microbiota-derived molecules in the development of heart disease and its risk factors has significantly increased attention towards the connection between our gut and heart. The gut microbiome is virtually an endocrine organ, arguably the largest, capable of contributing to and reacting to circulating signaling molecules within the host. Gut microbiota-host interactions occur through many pathways, including trimethylamine-N-oxide (TMAO) and short chain fatty acids (SCFA). These molecules and others have been linked to much pathology including chronic kidney disease, atherosclerosis, and hypertension.

Summary-Although our understanding of gut microbiota-host interactions has increased recently, many questions remain about the mechanistic links between the gut microbiome and CVD. With further research, we may one day be able to add gut microbiota profiles as an assessable risk factor for CVD and target therapies towards the gut microbiota.

\section{Keywords}

CVD; gut microbiome; TMAO; dysbiosis

\section{Introduction}

Cardiovascular disease (CVD) continues to be the leading cause of death globally and accounts for one of every three deaths in the United States [1]. Established risk factors for CVD include smoking, physical inactivity, obesity, diabetes mellitus, dyslipidemia, and

Address for correspondence: W. H. Wilson Tang, MD, 9500 Euclid Avenue, Desk J3-4, Cleveland, OH 44195, Phone: (216) 444-2121, Fax: (216) 445-6165, tangw @ ccf.org.

Disclosure: There are no relationships to disclose. 
hypertension. The recently discovered contribution of gut microbiota-derived molecules in the development of heart disease and its risk factors has significantly increased attention towards the connection between our gut and heart[2][3] and we may one day be able to add gut microbiota profiles as an assessable risk factor for CVD. The gut microbiome is virtually an endocrine organ, arguably the largest, capable of contributing to and reacting to circulating signaling molecules within the host. Thus, it is not surprising that dysbiosis, abnormal change in our gut flora, has been linked to a range of conditions, including obesity [4] and diabetes [5]. In this review, we will focus on the gut microbiome, its link to CVD, and potential therapeutic interventions (Figure 1).

\section{The Healthy Gut Microbiome}

The composition of a healthy individual's gut microbiome can vary remarkably from another's [6]; however, this composition is relatively stable over time [7]. The gut microbiome is primarily composed of species within the Bacteroidetes, Firmicutes, Actinobacteria, Proteobacteria, and Cerrucomicrobia phylae [8]. Over $90 \%$ of the total bacterial species in the healthy gut are Bacteroidetes and Firmicutes [8]. The relative abundance of species varies among individuals due to a variety of genetic and environmental factors, including diet and antibiotic use [6].

The gut microbiota has coevolved with us to serve a symbiotic role in extracting calories from otherwise indigestible macromolecules. Indigestible carbohydrates and proteins are fermented by colonic bacteria to form short chain fatty acids (SCFAs), volatile fatty acids containing fewer than six carbons. The most abundant SCFAs are acetic acid, propionic acid, and butyric acid. SCFAs are quickly and efficiently absorbed in the distal gut. SCFAs can be used in different biosynthetic routes by the host and also aid the host by reducing luminal $\mathrm{pH}$ [9]. Microorganisms that are incapable of catabolism of indigestible macromolecules are able to use SCFAs produced by other microorganisms as fuel in a process called metabolic cross-feeding [10].

In addition to being an energy source for both the host and microbiota, SCFAs are also signaling molecules which bind to G-protein coupled receptors GPR41 and GPR43 [11] known to be expressed in adipose tissue, intestines, and immune cells [12]. GPR43 receptors are essential for neutrophil recruitment, and the interaction between SCFAs and GPR43 is important in regulation of the inflammatory response [13]. As Maslowski et al. showed, both GPR43-deficient mice and germ-free mice (which are devoid of bacteria and thus have impaired SCFA production) showed increased production of inflammatory mediators [14]. SCFAs have been observed to reduce inflammatory cytokine production by inhibiting nuclear factor kappa B (NF- $\mathrm{BB})$ [15].

There is an important relationship between the gut microbiome, epithelial barrier, and the immune system. Intestinal epithelial cells detect bacteria and other microbes through Tolllike receptors (TLRs) and other Pattern Recognition Receptors; activation of these receptors allows activation of an immune response as well as pathways to sustain the gut barrier. This protects the host from systemic translocation of bacteria and allows bacteria to grow in the environment best suited for them—-the gut mucosa [16]. When the intestinal epithelium is 
activated to perform an immune function, its metabolic functions are reduced, and fat absorption and leptin levels have been observed to be reduced as a result [17]. The balance between metabolic functions and immune functions of the gut epithelium rests on the gut microbiome.

Commensal gut bacteria are also necessary for dampening an immune response to nonpathogenic bacteria, thus protecting the host from the harms of sterile inflammation. Diehl et al. showed that in antibiotic-induced dysbiotic mice, non-pathogenic Salmonella enterica was transported to the mesenteric lymph nodes and elicited a $\mathrm{T}$ cell response and IgA production, whereas in normal mice, the commensal bacteria prevented the unnecessary activation of the adaptive immune system [18].

Gut microbiota also affect host metabolism by altering the composition of bile acids. Colonic bacteria convert primary bile acids which have escaped reabsorption to secondary bile acids, which are less effective in emulsifying fat for absorption. A small amount of microbiota-derived secondary bile acids enter circulation and act as hormones, affecting signaling pathways involved in energy expenditure, metabolism, and inflammation [19].

\section{The Gut Microbiome and CVD}

A novel pathway between gut microbiota and CVD was described recently with the discovery of the link of trimethylamine-N-oxide (TMAO) to atherosclerosis [3].

Metabolomics first identified TMAO and choline as small-molecule metabolites associated with CVD risk in human plasma. Both molecules are derived from the metabolism of phosphatidylcholine. Phosphatidylcholine and other trimethylamine containing compounds such as L-carnitine, are metabolized by gut microbiota TMA lyases to produce the gas trimethylamine (TMA) which is metabolized by the host's liver enzymes of the Flavin monooxygenase (FMO) family to generate TMAO [20]. Mouse models confirmed that dietary supplementation of choline or TMAO increased TMAO levels, macrophage foam cell formation, and atherosclerotic development [3]. The enhanced atherosclerotic effects of phosphatidylcholine metabolism were shown to be dependent on gut microbiota as germfree conditions eliminated atherosclerosis development and dietary choline-derived TMAO [3]. L-carnitine diet supplementation showed similar effects [2].

In humans, a phosphatidylcholine challenge produced a post-prandial spike in circulating TMAO levels, and with suppression of intestinal microbiota by broad-spectrum antibiotics, there was a near-complete suppression of detectable TMAO [21]. In a large independent clinical cohort $(n=4,007)$, patients in the highest quartile of plasma TMAO levels had a 2.5 fold higher risk of a major adverse cardiovascular event than patients in the lowest quartile. Also, higher fasting plasma was found to predict the risk of incident major adverse cardiovascular events independent of traditional cardiovascular risk factors and the presence or extent of coronary artery disease [21].

TMAO has also been shown to enhance platelet hyperreactivity and thrombosis risk. Exposure of platelet rich plasma from healthy human volunteers to TMAO enhanced platelet activation from multiple agonists through augmented $\mathrm{Ca}(2+)$ release from intracellular 
stores. Mice on choline or TMAO supplemented diets showed enhanced thrombosis in contrast to germ-free mice with the same diet [22]. In a human cohort, there was a dosedependent association between plasma TMAO levels and platelet aggregation [23]. The directly observed effects of TMAO on atherosclerosis and platelet aggregation partially explain the increased risk of cardiovascular events with high TMAO levels.

Dysbiois has been linked to CVD and metabolic diseases, which are risk factors for CVD. In a study of 55 and 57 patients with the highest and lowest lifetime burdens of CVD risk factors, respectively, a change in microbiota profile was significantly associated with increased risk [24]. A lower ratio of Bacteroidetes to Firmicutes has been associated with obesity, and this proportion has been shown to increase with weight loss [25]. In hypertensive animals, dysbiosis in the form of decreased microbial diversity and decreased Bacteroidetes to Firmicutes ratio has been observed [26]. Collinsella has been found to be increased in patients with symptomatic atheroscleroris, defined as carotid stenosis leading to cerebrovascular events, compared to healthy controls who were enriched in Roseburia and Eubacterium [27]. In diet-induced obese mice, Akkermansia muciniphila abundance has been strongly inversely correlated with lipid metabolism and inflammation markers in adipose tissue [28].

Profiling gut microbiota of 531 well-phenotyped Finnish men from the Metabolic Syndrome In Men (METSIM) study revealed several associations between gut microbiota and the metabolic syndrome. Tenericutes and Christensenellaceae, which have been associated with lower BMI and triglyceride (TG) and higher HDL levels, were strongly associated with increased acetate, an SCFA, as were seven other unique taxa. Enrichment of Methanobrevibacter and Peptococcaceae were both also correlated with reduced TG levels. TMAO was directly associated with abundance of Peptococcaceae, Prevotella, and negatively with abundance of common gut commensal Faecalibacterium prausnitzii.

Given the gut microbiome's role in host metabolism, it is not surprising that it has also been implicated in development of obesity and diabetes, established risk factors for CVD [25]. SCFAs from gut microbiota have been shown to affect insulin sensitivity and suppress insulin-mediated fat accumulation [12]. SCFAs also regulate energy intake by stimulating the secretion of satiety hormones GLP1 and PPY [29] [30]. Administration of SCFAs without changing food intake or exercise lowered body weight and increased insulin sensitivity in mice on a high-fat diet [31].

Higher levels of TMAO have been found in diabetic animal models [32] as well as various disease patient cohorts [21] [33] [34] [35]. In patients with T2DM, TMAO levels portend higher adverse cardiovascular events and mortality risks independent of glycemic control [35]. Fecal microbiota transplants from lean donors to insulin-resistant individuals with metabolic syndrome has been shown to increase insulin sensitivity and the number of microbiota producing butyrate, an SCFA known to affect satiety hormones [36]. Miao et al. described a mechanistic connection between TMAO and diabetes with their finding that insulin downregulates FMO3 expression while glucagon does the opposite. Diabetic mouse models were shown to have elevated FMO3 expression and subsequently higher TMAO 
levels. Knockdown of elevated FMO3 improved insulin tolerance, hypercholesterolemia, and atherosclerosis, connecting the TMAO CVD and diabetes associations [37].

Hypertension has also been shown to interact with the gut microbiome; however, the mechanisms are largely unknown. SCFA signaling has been shown to reduce baseline blood pressure as GPR41 receptor-deficient mice were shown to have a systolic hypertensive phenotype [38]. In rats, TMAO infusion was found to enhance the hypertensive effects of angiotensin II [39]. Compared to conventionally raised mice, angiotensin II-infused germfree mice showed reduced endothelial dysfunction and showed other signs of systemic protection from angiotensin II-induced cardiovascular stress [40]. In a recent human study, $\mathrm{Li}$ et al. found decreased microbial richness and diversity in pre-hypertensive and hypertensive populations; fecal transplantation from hypertensive individuals to germ-free mice resulted in elevated blood pressure, demonstrating a direct effect [41].

Dysbiosis has also been observed in kidney diseases, which are closely related to CVD [42]. In 521 stable subjects with chronic kidney disease (CKD), TMAO levels were elevated and predictive of poorer long-term survival after adjustment for traditional risk factors [43]. DNA of gut microbiota has been found in systemic circulation of patients with CKD on hemodialysis [44], indicating a disruption of the intestinal epithelial barrier. Suppression of gut microbiota with antibiotics was shown to reduce serum endotoxin levels and reverse systemic inflammation in a CKD mouse model [45].

Bacterial dysbiosis can lead to overproduction of nitrogenous compounds which promote disruption of intestinal epithelial tight junctions. Disruption of the intestinal epithelium leads to translocation of gut bacterial DNA and uremic toxins into circulation.

Lipopolysacchardies and uremic toxins bind to Pattern Recognition Receptors including Toll-like Receptors (TLRs), activating the inflammatory pathway. Systemic inflammation is thought to underlie a variety of disease, including CVD and CKD. Atherosclerotic plaques have been shown to include bacterial DNA, mostly of Proteobacteria, and the same bacteria found in atherosclerotic plaques have been found in the same individuals' gut [46], suggesting a disruption in the intestinal epithelial barrier. Disruption of intestinal epithelial tight junctions is thought to be caused at least partially by ammonia and ammonium hydroxide [42]. Gut microbiota contribute to ammonia and ammonium hydroxide production by hydrolyzing urea. Ammonium can also be converted to nitrite and nitric oxide by microbiota. Gut microbiota also produce nitric oxide from nitrite in saliva produced by facultative anaerobic bacteria in the oral cavity through reduction of ingested nitrate [47].

\section{Therapeutic Intervention}

The discovery of the TMAO pathway and evidence for links between gut microbiota and several risk factors for CVD offers new opportunities for therapeutic interventions. With alterations in the gut microbial community being associated with much pathology, interventions aimed at changing the microbial composition present a promising therapeutic approach. A high fiber diet has been reported to increase SCFA-producing microbiota and lower blood pressure in a portion of end stage renal disease patients [48]. Intake of prebiotics, which are typically food indigestible molecules eliciting a favorable impact on 
gut microbiome composition, can also be beneficial. In an insulin resistant mouse model, prebiotics increased Bacteroidetes to Firmicutes ratio and reduced other characteristics associated with the diabetic phenotype [49]. However, the effects of prebiotic treatment have been inconsistent across human studies, which may partially be due to inconsistencies in defining prebiotic treatment. The same is true for probiotics, which are compilations of live bacteria administered to promote gut microbiome health, most commonly Lactobacilli and Bifidobacteria. Administration of specific bacteria has been shown to partially protect mice from diet-induced obesity, increase insulin secretion, and reduce cholesterol [50]. Prebiotic feeding has also been shown to normalize Akkermansia muciniphil, which has been shown to be decreased in high-fat diet-induced metabolic disorders [51].

While probiotics and prebiotics are focused on increasing beneficial bacteria, antibiotic treatment is focused on reducing harmful bacteria content. However, the lack of specificity of antibiotics prevents targeting of specific bacterial populations. Given the uniqueness of each individual's gut microbial community and the lack of knowledge on the contributions of specific strains to CVD, a more targeted approach is warranted.

Fecal microbiota transplantation (FMT) is designed to replace an individual's gut microbiota with that of a healthy donor's, ideally displacing pathogenic strains. FMT offers an effective treatment option for Clostridium difficile infection in humans as well as an investigative new treatment for other gastrointestinal disorders such as IBD. FMT has not only been used to study the links between gut microbiota and CVD but also in treatment of cardiometabolic disorders. Allogenic fecal transplant has been shown to improve insulin sensitivity independent of weight variations compared to autologous transfer [36]. Although this initial success in treating the obesity aspect of CVD has been promising, other aspects need to be studied as well as methods for reducing complications from the procedure.

With the discovery of the TMAO pathway linking the gut microbiome and CVD, we are presented with a new opportunity for CVD treatment and prevention. Recently, a structural analog of choline, 3,3-dimethyl-1-butanol (DMB), was shown to inhibit TMA production and reduce circulating TMAO levels in mice on a high choline or L-carnitine diet, which reduced macrophage foam cell formation and atherosclerosis in vivo [52]. Importantly, this small-molecule inhibitor is not lethal to the bacterium, allowing for specific targeting of the link between gut microbiota and CVD and reduced selective pressure for development of resistance.

\section{Summary}

Dysbiosis has been implicated in CVD as well as many aspects of cardiometabolic syndrome: obesity, hypertension, chronic kidney disease, and diabetes. A mechanistic link between gut microbiota formation of TMAO to CVD has been demonstrated in various animal and human studies. TMAO has been implicated in atherosclerosis, platelet aggregation, diabetes, and hypertension. Therapies targeting the gut microbiota, including fecal microbiota transplantation (FMT), pre/probiotics, and TMAO inhibitors, present new opportunities for CVD treatment. 


\section{Acknowledgments}

Dr. Tang is funded by research grant support from the National Institutes of Health and the Office of Dietary Supplements (P20HL113452, R01DK106000, R01HL126827).

\section{References}

1. Writing Group Members. Heart Disease and Stroke Statistics-2016 Update: A Report From the American Heart Association. Circulation. Jan; 2016 133(4):e38-360. [PubMed: 26673558]

$2 * *$. Koeth RA, et al. Intestinal microbiota metabolism of 1-carnitine, a nutrient in red meat, promotes atherosclerosis. Nat Med. May; 2013 19(5):576-585. This paper first identified TMAO as the link between a high red meat diet and atherosclerosis, a formative event in the continued discovery of the link between the gut and the heart. [PubMed: 23563705]

3. Wang Z, et al. Gut flora metabolism of phosphatidylcholine promotes cardiovascular disease. Nature. Apr; 2011 472(7341):57-63. [PubMed: 21475195]

4. Turnbaugh PJ, Ley RE, Mahowald MA, Magrini V, Mardis ER, Gordon JI. An obesity-associated gut microbiome with increased capacity for energy harvest. Nature. Dec; 2006 444(7122):1027131. [PubMed: 17183312]

5. Qin J, et al. A metagenome-wide association study of gut microbiota in type 2 diabetes. Nature. Oct; 2012 490(7418):55-60. [PubMed: 23023125]

$6 * *$. Consortium THMP. Structure, function and diversity of the healthy human microbiome. Nature. Jun; 2012 486(7402):207-214. This systematic investigation of the gut microbiome gives key insight to its composition. [PubMed: 22699609]

7. Faith JJ, et al. The Long-Term Stability of the Human Gut Microbiota. Science. Jul.2013 341(6141): 1237439. [PubMed: 23828941]

8. Qin J, et al. A human gut microbial gene catalogue established by metagenomic sequencing. Nature. Mar; 2010 464(7285):59-65. [PubMed: 20203603]

9. Ríos-Covián D, Ruas-Madiedo P, Margolles A, Gueimonde M, de los Reyes-Gavilán CG, Salazar N. Intestinal Short Chain Fatty Acids and their Link with Diet and Human Health. Front Microbiol. 2016; 7

10. den Besten G, et al. Gut-derived short-chain fatty acids are vividly assimilated into host carbohydrates and lipids. Am J Physiol - Gastrointest Liver Physiol. Dec; 2013 305(12):G900G910. [PubMed: 24136789]

$11^{* *}$. Brown AJ, et al. The Orphan G protein-coupled receptors GPR41 and GPR43 are activated by propionate and other short chain carboxylic acids. J Biol Chem. Mar; 2003 278(13):1131211319. This paper shows the molecular signaling of SCFAs through GPR41 and GPR43, receptors which were never before tied to any specific function. [PubMed: 12496283]

12. Kimura I, et al. The gut microbiota suppresses insulin-mediated fat accumulation via the shortchain fatty acid receptor GPR43. Nat Commun. May.2013 4:1829. [PubMed: 23652017]

13. Sina $\mathrm{C}$, et al. G protein-coupled receptor 43 is essential for neutrophil recruitment during intestinal inflammation. J Immunol Baltim Md 1950. Dec; 2009 183(11):7514-7522.

14. Maslowski KM, et al. Regulation of inflammatory responses by gut microbiota and chemoattractant receptor GPR43. Nature. Oct; 2009 461(7268):1282-1286. [PubMed: 19865172]

15. Usami M, et al. Butyrate and trichostatin A attenuate nuclear factor kappaB activation and tumor necrosis factor alpha secretion and increase prostaglandin E2 secretion in human peripheral blood mononuclear cells. Nutr Res N Y N. May; 2008 28(5):321-328.

16. Cerf-Bensussan N, Gaboriau-Routhiau V. The immune system and the gut microbiota: friends or foes? Nat Rev Immunol. Oct; 2010 10(10):735-744. [PubMed: 20865020]

$17 * *$. Shulzhenko N, et al. Crosstalk between B lymphocytes, microbiota and the intestinal epithelium governs immunity versus metabolism in the gut. Nat Med. Dec; 2011 17(12):1585-1593. This paper presented a new way to think about the balance between metabolism and immunity functions of the intestinal epithelium and the role of gut microbiota within that. [PubMed: 22101768] 
18**. Diehl GE, et al. Microbiota restricts trafficking of bacteria to mesenteric lymph nodes by CX3CR1hi cells. Nature. Feb; 2013 494(7435):116-120. This paper was influential for clearly demonstrating the role of commensal gut microbiota in suppressing immune activation to nonpathogenic bacteria. [PubMed: 23334413]

19. Chiang JYL. Bile acid metabolism and signaling. Compr Physiol. Jul; 2013 3(3):1191-1212. [PubMed: 23897684]

20. Bennett BJ, et al. Trimethylamine-N-oxide, a metabolite associated with atherosclerosis, exhibits complex genetic and dietary regulation. Cell Metab. Jan; 2013 17(1):49-60. [PubMed: 23312283]

$21^{* *}$. Tang WHW, et al. Intestinal Microbial Metabolism of Phosphatidylcholine and Cardiovascular Risk. N Engl J Med. Apr; 2013 368(17):1575-1584. In the first study of TMAO's link to CVD risk in a human cohort, TMAO was shown to be independently predictive of adverse cardiovascular events. [PubMed: 23614584]

22. Zhu W, et al. Gut Microbial Metabolite TMAO Enhances Platelet Hyperreactivity and Thrombosis Risk. Cell. Mar; 2016 165(1):111-124. [PubMed: 26972052]

23**. Zhu W, Wang Z, Tang WHW, Hazen SL. Gut Microbe-Generated Trimethylamine N-Oxide From Dietary Choline Is Prothrombotic in Subjects. Circulation. Apr; 2017 135(17):1671-1673. In this recent study, TMAO was found to enhance platelet reactivity and thrombus formation in humans. The combination of this finding and others on atherosclerosis helps explains the increased risk for adverse cardiovascular events. [PubMed: 28438808]

24. Kelly TN, et al. Gut Microbiome Associates With Lifetime Cardiovascular Disease Risk Profile Among Bogalusa Heart Study Participants. Circ Res. Sep; 2016 119(8):956-964. [PubMed: 27507222]

25. Ley RE, Turnbaugh PJ, Klein S, Gordon JI. Microbial ecology: human gut microbes associated with obesity. Nature. Dec; 2006 444(7122):1022-1023. [PubMed: 17183309]

26. Yang T, et al. Gut dysbiosis is linked to hypertension. Hypertens Dallas Tex 1979. Jun; 2015 65(6): 1331-1340.

27. Karlsson FH, et al. Symptomatic atherosclerosis is associated with an altered gut metagenome. Nat Commun. Dec.2012 3:1245. [PubMed: 23212374]

28. Schneeberger M, et al. Akkermansia muciniphila inversely correlates with the onset of inflammation, altered adipose tissue metabolism and metabolic disorders during obesity in mice. Sci Rep. Nov.2015 5:16643. [PubMed: 26563823]

29. Psichas A, et al. The short chain fatty acid propionate stimulates GLP-1 and PYY secretion via free fatty acid receptor 2 in rodents. Int J Obes 2005. Mar; 2015 39(3):424-429.

30. Tolhurst G, et al. Short-chain fatty acids stimulate glucagon-like peptide-1 secretion via the Gprotein-coupled receptor FFAR2. Diabetes. Feb; 2012 61(2):364-371. [PubMed: 22190648]

31. den Besten G, et al. Short-Chain Fatty Acids Protect Against High-Fat Diet-Induced Obesity via a PPAR $\gamma$-Dependent Switch From Lipogenesis to Fat Oxidation. Diabetes. Jul; 2015 64(7):23982408. [PubMed: 25695945]

32. Dambrova M. Diabetes is Associated with Higher Trimethylamine N-oxide Plasma Levels. Exp Clin Endocrinol Diabetes Off J Ger Soc Endocrinol Ger Diabetes Assoc. Apr; 2016 124(4):251256.

33. Tang WHW, et al. Intestinal microbiota-dependent phosphatidylcholine metabolites, diastolic dysfunction, and adverse clinical outcomes in chronic systolic heart failure. J Card Fail. Feb; 2015 21(2):91-96. [PubMed: 25459686]

34. Tang, WH Wilson, et al. Prognostic Value of Elevated Levels of Intestinal Microbe-Generated Metabolite Trimethylamine-N-oxide in Patients with Heart Failure: Refining the Gut Hypothesis. J Am Coll Cardiol. Nov; 2014 64(18):1908-1914. [PubMed: 25444145]

35. Tang WHW, et al. Increased Trimethylamine N-Oxide Portends High Mortality Risk Independent of Glycemic Control in Patients with Type 2 Diabetes Mellitus. Clin Chem. Jan; 2017 63(1):297306. [PubMed: 27864387]

36. Vrieze A, et al. Transfer of intestinal microbiota from lean donors increases insulin sensitivity in individuals with metabolic syndrome. Gastroenterology. Oct; 2012 143(4):913-916.e7. [PubMed: 22728514] 
37. Miao J, et al. Flavin-containing monooxygenase 3 as a potential player in diabetes-associated atherosclerosis. Nat Commun. Apr.2015 6:6498. [PubMed: 25849138]

$38 * *$. Natarajan N, et al. Microbial short chain fatty acid metabolites lower blood pressure via endothelial G-protein coupled receptor 41. Physiol Genomics. Sep.2016 doi:physiolgenomics. 00089.2016. This paper clearly demonstrates the effects of gut-microbiota derived SCFAs on blood pressure.

39. Ufnal M, Jazwiec R, Dadlez M, Drapala A, Sikora M, Skrzypecki J. Trimethylamine-N-Oxide: A Carnitine-Derived Metabolite That Prolongs the Hypertensive Effect of Angiotensin II in Rats. Can J Cardiol. Dec; 2014 30(12):1700-1705. [PubMed: 25475471]

40. Karbach SH, et al. Gut Microbiota Promote Angiotensin II-Induced Arterial Hypertension and Vascular Dysfunction. J Am Heart Assoc. Sep.2016 5(9)

41. Li J, et al. Gut microbiota dysbiosis contributes to the development of hypertension. Microbiome. Feb.2017 5(1):14. [PubMed: 28143587]

42. Vaziri ND, et al. Chronic kidney disease alters intestinal microbial flora. Kidney Int. Feb; 2013 83(2):308-315. [PubMed: 22992469]

43**. Tang WHW, et al. Gut Microbiota-Dependent Trimethylamine N-Oxide (TMAO) Pathway Contributes to Both Development of Renal Insufficiency and Mortality Risk in Chronic Kidney DiseaseNovelty and Significance. Circ Res. Jan; 2015 116(3):448-455. In a human cohort, TMAO was found to contribute to CKD and the overall cardiorenal syndrome. [PubMed: 25599331]

44. Shi K, et al. Gut bacterial translocation may aggravate microinflammation in hemodialysis patients. Dig Dis Sci. Sep; 2014 59(9):2109-2117. [PubMed: 24828917]

45. Andersen K, et al. Intestinal Dysbiosis, Barrier Dysfunction, and Bacterial Translocation Account for CKD-Related Systemic Inflammation. J Am Soc Nephrol JASN. Jan; 2017 28(1):76-83. [PubMed: 27151924]

46**. Koren O, et al. Human oral, gut, and plaque microbiota in patients with atherosclerosis. Proc Natl Acad Sci U S A. Mar; 2011 108(Suppl 1):4592-4598. This study provided strong implications for gut barrier dysfunction in atherosclerosis. [PubMed: 20937873]

47. Briskey D, Tucker PS, Johnson DW, Coombes JS. Microbiota and the nitrogen cycle: Implications in the development and progression of CVD and CKD. Nitric Oxide. Jul.2016 57:64-70. [PubMed: 27164294]

48. Marques FZ, et al. High-Fiber Diet and Acetate Supplementation Change the Gut Microbiota and Prevent the Development of Hypertension and Heart Failure in Hypertensive MiceClinical Perspective. Circulation. Mar; 2017 135(10):964-977. [PubMed: 27927713]

49. Everard A, et al. Responses of gut microbiota and glucose and lipid metabolism to prebiotics in genetic obese and diet-induced leptin-resistant mice. Diabetes. Nov; 2011 60(11):2775-2786. [PubMed: 21933985]

50. Tuohy KM, Fava F, Viola R. 'The way to a man's heart is through his gut microbiota'--dietary proand prebiotics for the management of cardiovascular risk. Proc Nutr Soc. May; 2014 73(2):172185. [PubMed: 24495527]

51. Everard A, et al. Cross-talk between Akkermansia muciniphila and intestinal epithelium controls diet-induced obesity. Proc Natl Acad Sci. May; 2013 110(22):9066-9071. [PubMed: 23671105]

52. Wang Z, et al. Non-lethal Inhibition of Gut Microbial Trimethylamine Production for the Treatment of Atherosclerosis. Cell. Dec; 2015 163(7):1585-1595. [PubMed: 26687352] 


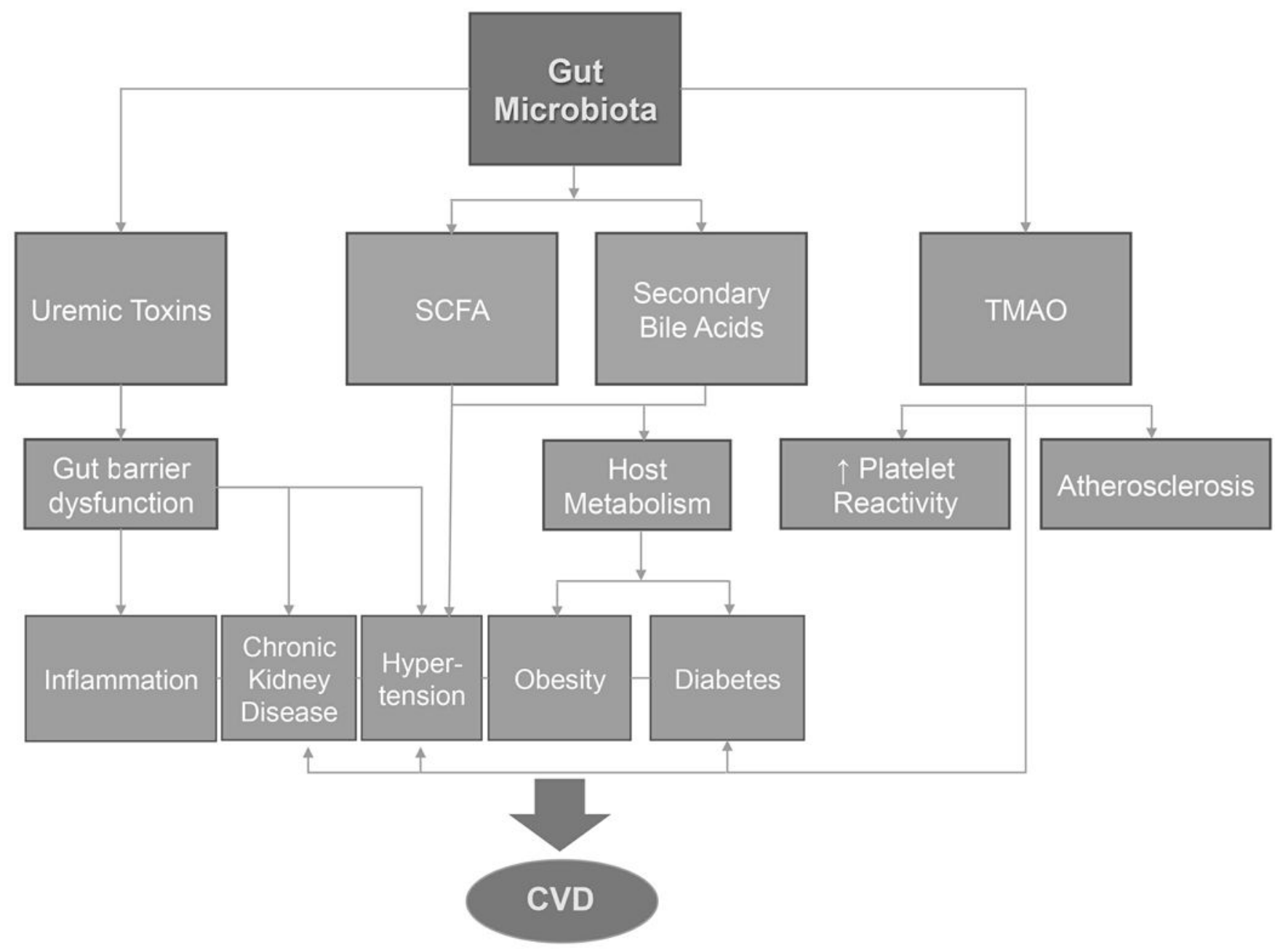

Figure 1.

Schematic of gut microbiota effects on risk factors for CVD and adverse cardiovascular events. 